\title{
Unstable angina in a young woman with Hodgkin's lymphoma
}

\author{
Paweł Krzesiński, Katarzyna Piotrowicz, Grzegorz Jan Horszczaruk, Jarosław Kowal
}

Department of Cardiology and Internal Diseases, Military Institute of Medicine, Warsaw, Poland

\begin{abstract}
We present the case of a 30 year-old woman - 16 months after successful treatment of Hodgkin's lymphoma (chest location) with chemotherapy (including doxorubicin) and radiotherapy - with recurrent chest pain and dyspnea. In ambulatory event telemetry, she reported nocturnal chest pain with transient ST elevation characteristic for acute cardiac ischemia. Urgent coronary angiography with intravascular ultrasound and virtual histology visualization revealed $70 \%$ fibrotic stenosis in the proximal segment of the left anterior descending artery successfully treated with a drug-eluting stent implantation. This case is especially noteworthy because of the short period from initial therapy to the symptomatic coronary artery disease that is entirely unlike the reported mean latency period of several years. (Cardiol J 2013; 20, 1: 90-93)
\end{abstract}

Key words: coronary artery disease, acute coronary syndrome, radiotherapy, chemotherapy, anthracyclines

\section{Introduction}

Long-term mortality after the successful treatment of Hodgkin's lymphoma (HL) is higher than in the general population, especially on account of cardiac events. The risk factors of cardiovascular complications are: irradiation of the chest field, high dose of radiation (> $30 \mathrm{~Gy}$ ), anthracycline therapy, exposure at young age, and time after initial treatment. Such patients might suffer from early coronary disease, myocardial infarction, valvular disease, conduction abnormalities, arrhythmias and sudden cardiac death. Radiation-induced myocardial injury provokes endothelial dysfunction, microvascular insufficiency, direct myocite toxicity, and fibrosis. Moreover, the anthracycline therapy (especially with high doses of doxorubicin) induces heart fibrosis and cardiomyocyte death [1-3]. Although this pathogenesis is well proved, there remains insufficient awareness of the problem among cardiologists, oncologists and primary care physicians.

\section{Case presentation}

A 30 year-old woman was referred for cardiology consultation to the Department of Cardiology and Internal Diseases of the Military Institute of Medicine due to recurrent chest pain and dyspnea. Roughly 16 months previously, she had undergone successful treatment of HL (chest location) with chemotherapy (doxorubicin, bleomycin, vinblastine, and dacarbazine) and radiotherapy (RT, total $36 \mathrm{~Gy}$ ). She was a non-smoker, non-obese, and with no family history of coronary artery disease (CAD). After ambulatory consultation by a general practitioner, an oncologist and a cardiologist, it was suspected that there was a psychogenic background to the described symptoms. A 12-lead electrocardiogram (ECG) and echocardiography showed no abnormalities. Laboratory findings displayed hyperlipidemia (total cholesterol of $239 \mathrm{mg} / \mathrm{dL}$, LDL $165 \mathrm{mg} / \mathrm{dL}$, HDL $46 \mathrm{mg} / \mathrm{dL}$, triglycerides $141 \mathrm{mg} / \mathrm{dL})$. Long-term monitoring with a real-time interpretation of ECG revealed ventricu-

Address for correspondence: Paweł Krzesiński, PhD, Department of Cardiology and Internal Diseases, Military Institute of Medicine, ul. Szaserów 128, 04-141 Warszawa 44, Poland, tel: +48 22681 63 89, fax: +48 228108089 ,

e-mail: pkrzesinski@wim.mil.pl 


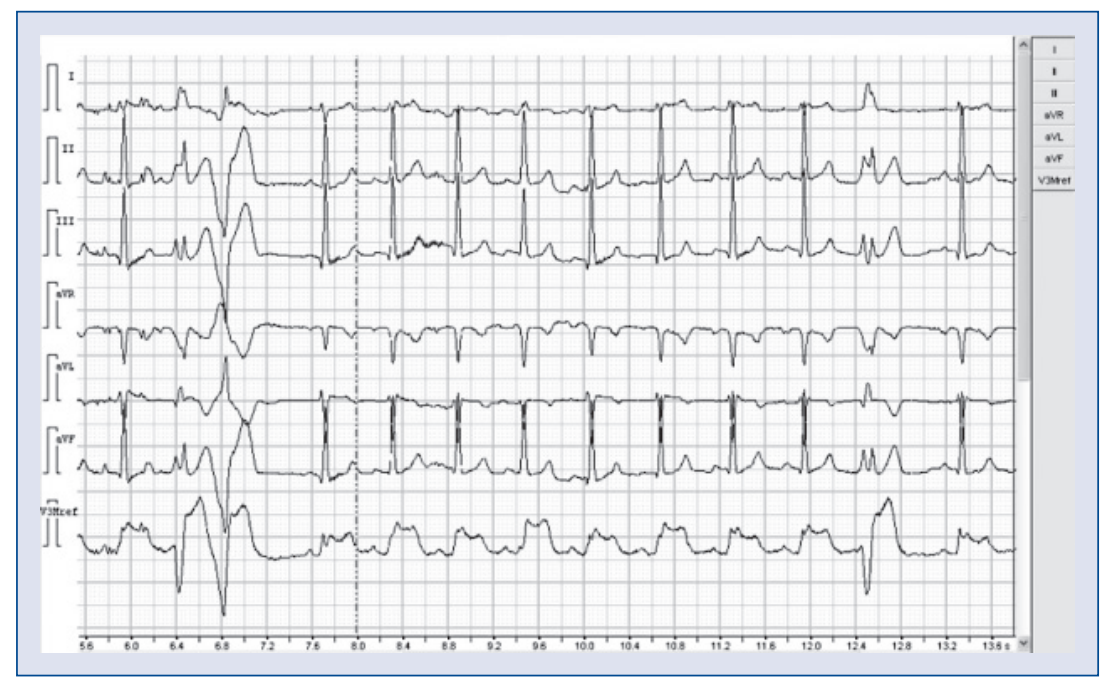

Figure 1. Transient ST elevation in V3 lead (telemetric ECG) and single ventricular ectopic beats.

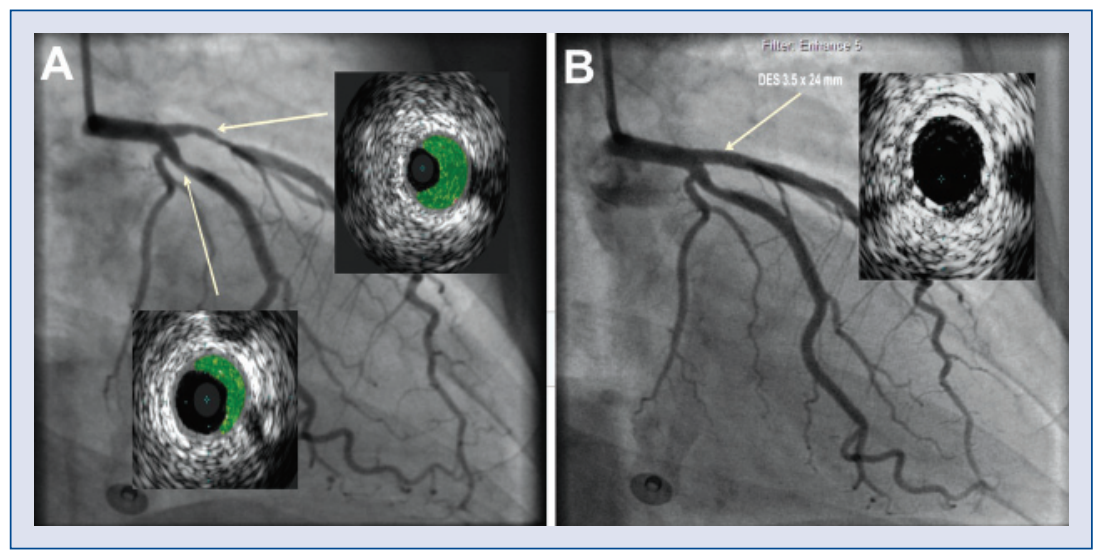

Figure 2. Angiography, intravascular ultrasound (IVUS) and virtual histology (VH). A. Left coronary artery before percutaneous coronary intervention: eccentric lesion in LAD: MLA $2.9 \mathrm{~mm}^{2}$, LAS 65\%, MLD $1.9 \mathrm{~mm}$, plaque burden at MLD 77\%; VH: mainly fibrotic (green color) ca. $76 \%$ vol. Eccentric lesion in obtuse marginal branch: MLA $5.4 \mathrm{~mm}^{2}$, LAS 45\%; MLD $2.4 \mathrm{~mm}$, plaque burden at MLD 50\%, VH: mainly fibrotic (82\% vol.); B. Left coronary artery post percutaneous coronary intervention: stent in LAD fully apposed with good expansion: MLA $9.3 \mathrm{~mm}^{2}$; LAD — left anterior descending artery; LAS — luminal area stenosis; MLA — minimal luminal cross-sectional area; MLD - minimal luminal diameter.

lar ectopic beats, an episode of nonsustained ventricular tachycardia, and ST depressions and elevations.

The patient was recruited to ambulatory event telemetry, and within a few days reported nocturnal chest pain with transient ST elevation in V3 lead (Fig. 1). Urgent coronary angiography revealed $70 \%$ stenosis in the proximal segment of the left anterior descending artery (LAD), and $40 \%$ stenosis in the obtuse marginal branch (OM). Intravascular ultrasound (IVUS) confirmed the presence of eccentric lesions in $\mathrm{LAD}$ and $\mathrm{OM}$; the lesion in the LAD was significant based on IVUS criteria (Fig. 2A). As assessed by virtual histology (VH), both lesions were mainly fibrotic. The patient was successfully treated with a drug-eluting stent (Taxus Liberte $3.5 \times 24 \mathrm{~mm}$ ) in LAD (Fig. 2B). Applied pharmacotherapy involved: clopidogrel, acetylsalicylic acid, atorvastatin, verapamil, ramipril, and trimetazidin. At 11 months follow-up, she reported no symptoms.

\section{Discussion}

An increased risk of $\mathrm{CAD}$ is a well-known longterm complication after mediastinal RT for HL. 
Symptomatic cardiac disease after RT occurs in about $10 \%$ of patients, but many of them develop subclinical histological changes [2, 4]. This becomes an important clinical problem as approximately $50 \%$ of HL patients may require RT [5]. The manifestations include not only CAD but also arrhythmia, acute pericarditis, pericardial effusion with or without cardiac tamponade, and constructive pericarditis [6].

In the reported case, a short period from initial therapy (about 16 months) to the symptomatic $\mathrm{CAD}$ is very unlike the lengthy mean latency period that has been reported before [2]. Reinders et al. [1] revealed that the curve of cardiovascular (CV) risk was almost flat until $4-5$ years after RT, and increased rapidly after ten years (risk for ischemic cardiac death at 10 years $-2.6 \%$, at 25 years $10.2 \%)$. In a cohort of 1,271 patients, Strasser et al. [7] observed significantly higher CV risk in patients after RT but not within the first few years. Among 294 patients with HL screened for CV disease by Heidenreich et al. [3], stress-induced ECG changes were observed in only three of 88 patients $2-$ -10 years after RT. Of these, only one had ostial disease $\geq 50 \%$, and none was of more than one vessel disease. In view of these studies, the rapid progression of atherosclerosis in our case seems indeed to be unique.

This might be caused by an accumulation of the risk factors mentioned above (young age, irradiation of the chest field, high dose of radiation, doxorubicin therapy). Although the global risk is highest in patients with typical atherogenic factors (age over 50 years, family history of $\mathrm{CAD}$, hypertension, smoking, obesity, hyperlipidemia or diabetes), patients treated for HL at a younger age are - in comparison with the general population of the same age - at relatively higher risk of cardiac disease than older patients [8].

The treatment with doxorubicin - reported in this case - can substantially contribute to higher $\mathrm{CV}$ risk. Myrehaug et al. [9] reported that treatment with both anthracycline and radiation is associated with a significantly increased risk of cardiac events (RR 2.8). The risk was lower for patients given only mediastinal irradiation (RR 1.9). Aleman et al. [10] also revealed that anthracycline-based chemotherapy increased the risk of heart failure (RR 2.8) and valvular disease (RR 2.1). These specific risk factors of HL therapy should be carefully screened, especially in younger patients.

The coronary lesions in patients with HL have some characteristic features. One of them is the location of atherosclerotic plaques. In the presented case, coronary angiography revealed stenosis in the proximal segments of large coronary arteries, included in the radiation field. The arteries located in the anterior of the chest are more exposed to radiation and are the most frequent location of the stenotic lesions in patients with HL $[1,11]$.

Radiation-induced atherosclerosis also has its specific histological manifestation. In this woman, VH revealed mainly fibrotic lesions. The pathogenesis of such injury is complex. The RT induces endothelial damage, perivascular fibrosis and intimal thickening. It provokes smooth muscle cells proliferation, collagen intimal and medial deposits, and an increase in the number of plasma cells with the presence of large fibroblasts $[11,12]$. This is the opposite of typical atherosclerotic plaques that are more abundant in lipids and lymphocytes [6] and with a higher risk of rupture. On the other hand, in vitro studies have revealed the prothrombotic effect of radiation connected with higher release of von Willebrand factor that can provoke coronary thrombosis [13].

The choice of angioplasty with drug-eluting stent implantation was in the presented case based on current guidelines. However, even in multi-vessel coronary disease, percutaneous coronary revascularization is the option of first choice in the management even of complex lesions because the performance of coronary artery bypass grafting can encounter some difficulties. The main potential surgical problem is post-irradiation mediastinal fibrosis. Accelerated atherosclerosis of internal mammary arteries should also be taken into consideration $[2,11,14]$.

Our case makes the argument for early cardiac monitoring of even asymptomatic survivors of HL. Oncologists and primary care physicians should educate these patients as to the potential latent adverse effects of the therapy, especially CV complications. Screening for CAD by using noninvasive methods such as stress echocardiography, stress radionuclide perfusion imaging, and computed tomography with coronary artery calcium score quantification, is suggested [3, 15]. As a group at risk of premature, and significantly accelerated, atherosclerosis, these patients should be treated comprehensively and carefully monitored for $\mathrm{CAD}$ progression. The presented case justifies even earlier CV diagnostics than the five years after RT recommended by Heidenreich et al. [3].

\section{Conclusions}

Cardiovascular complications after radio- and chemotherapy may occur in young survivors of HL. 
Even in the absence of typical CV risk factors, they demand an especially careful approach, and any atypical symptoms should not be ignored. General awareness of early cardiac complications after oncotherapy should prevent delayed diagnosis and inappropriate treatment of these patients.

Conflict of interest: Pawel Krzesiński and Jarosław Kowal received a consultancy fee for performing the ambulatory event telemetry from the National Institute of Cardiology as part of the 'Establishment of TeleInterMed Teleconsulting Center' project at the Stefan Cardinal Wyszyński National Institute of Cardiology, supported by a grant from the European Economic Area Financial Mechanism (no PL 0060). The other authors declare no conflict of interest.

\section{References}

1. Reinders JG, Heijmen BJ, Olofsen-van Acht MJ, van Putten WL, Levendag PC. Ischemic heart disease after mantlefield irradiation for Hodgkin's disease in long-term follow-up. Radiother Oncol, 1999; 51: 35-42.

2. Lee PJ, Mallik R. Cardiovascular effects of radiation therapy: Practical approach to radiation therapy-induced heart disease. Cardiol Rev, 2005; 13: 80-86.

3. Heidenreich PA, Schnittger I, Strauss HW et al. Screening for coronary artery disease after mediastinal irradiation for Hodgkin's disease. J Clin Oncol, 2007; 25: 43-49.

4. Friedlander AH, Sung EC, Child JS. Radiation-induced heart disease after Hodgkin's disease and breast cancer treatment: Dental implications. J Am Dent Assoc, 2003; 134: 1615-1620.
5. Galper SL, Yu JB, Mauch PM et al. Clinically significant cardiac disease in patients with Hodgkin lymphoma treated with mediastinal irradiation. Blood, 2011; 117: 412-418.

6. Mert M, Arat-Ozkan A, Ozkara A, Aydemir NA, Babalik E. Radiation-induced coronary artery disease. Z Kardiol, 2003; 92: 682-685 .

7. Strasser JF, Li S, Neuberg D, Silver B, Ng AK, Mauch PM. Late cardiac toxicity after mediastinal radiation therapy for Hodgkin's disease. Int J Radiat Oncol Biol Phys, 2004; 60: S217-S218.

8. Hancock SL, Tucker MA, Hoppe RT. Factors affecting late mortality from heart disease after treatment of Hodgkin's disease. JAMA, 1993; 270: 1949-1955.

9. Myrehaug S, Pintilie M, Tsang R et al. Cardiac morbidity following modern treatment for Hodgkin lymphoma: Supra-additive cardiotoxicity of doxorubicin and radiation therapy. Leuk Lymphoma, 2008; 49: 1486-1493.

10. Aleman BM, van den Belt-Dusebout AW, De Bruin ML et al. Late cardiotoxicity after treatment for Hodgkin lymphoma. Blood, 2007; 109: 1878-1886.

11. Jurcut R, Savu O, Giusca S, Deleanu D, Ciudin R, Ginghina C. Between Scylla and Charybdis: Long-term cardiovascular complications after radiotherapy for Hodgkin's lymphoma. Hellenic J Cardiol, 2009; 50: 538-543.

12. van Rijswijk S, Huijbregts MA, Lust E, Strack van Schijndel RJ. Mini-review on cardiac complications after mediastinal irradiation for Hodgkin lymphoma. Neth J Med, 2008; 66: 234-237.

13. Sebag-Montefiore D, Hope-Stone H. Radiation induced coronary heart disease. Br Heart J, 1993; 69: 481-482.

14. Renner SM, Massel D, Moon BC. Mediastinal irradiation. A risk factor for atherosclerosis of the internal thoracic arteries. Can J Cardiol, 1999; 15: 597-600.

15. Andersen R, Wethal T, Günther A et al. Relation of coronary artery calcium score to premature coronary artery disease in survivors > 15 years of Hodgkin's lymphoma. Am J Cardiol, 2010; 105: 149-152. 